\title{
New properties of the generalized Dini function
}

\author{
Ebrahim Analouei Adegani*1 (D), Teodor Bulboacă ${ }^{2}$ (D) \\ ${ }^{1}$ Faculty of Mathematical Sciences, Shahrood University of Technology, P.O.Box 316-36155, Shahrood, \\ Iran \\ ${ }^{2}$ Faculty of Mathematics and Computer Science, Babeş-Bolyai University, 400084 Cluj-Napoca, Romania
}

\begin{abstract}
In this work we study some properties of the normalized form of generalized Dini function like close-to-convexity of some order and close-to-convex with respect to another convex function. Furthermore, we investigate sufficient conditions which these functions are uniformly $k$-starlike functions of complex order $b$ in the open unit disk, and some consequences of the main results are also presented.
\end{abstract}

Mathematics Subject Classification (2010). 33E12, 30C45

Keywords. analytic functions, univalent function, Bessel functions of first kind of order $\nu$, modified Dini function, starlike, convex, and close-to-convex functions of order $\alpha$

\section{Introduction and preliminaries}

Let $\mathcal{A}$ be the class of functions of the form

$$
f(z)=z+\sum_{n=2}^{\infty} a_{n} z^{n}
$$

which are analytic in the open unit disk $\mathbb{U}:=\{z \in \mathbb{C}:|z|<1\}$, and let $\mathcal{S}$ denote the class of all functions of $\mathcal{A}$ which are univalent in $\mathbb{U}$.

Furthermore, let $\mathcal{P}(\gamma)$ denote the class of all analytic functions consisting of functions $p$ with $p(0)=1$ such that

$$
\operatorname{Re} p(z)>\gamma, z \in \mathbb{U},(0 \leq \gamma<1),
$$

and in particular, $\mathcal{P}:=\mathcal{P}(0)$ is the well-known Caratheódory class of functions with positive real part in $\mathbb{U}$.

We denote by $\mathcal{S}^{*}(\alpha)$ and $\mathcal{C}(\alpha)$ the subclasses of $\mathcal{A}$ consisting of functions which are starlike of order $\alpha$, and convex of order $\alpha$, that is

$$
\mathcal{S}^{*}(\alpha):=\left\{f \in \mathcal{A}: \operatorname{Re} \frac{z f^{\prime}(z)}{f(z)}>\alpha, z \in \mathbb{U}\right\},(0 \leq \alpha<1)
$$

and

$$
\mathcal{C}(\alpha):=\left\{f \in \mathcal{A}: \operatorname{Re} \frac{\left(z f^{\prime}(z)\right)^{\prime}}{f^{\prime}(z)}>\alpha, z \in \mathbb{U}\right\},(0 \leq \alpha<1)
$$

*Corresponding Author.

Email addresses: analoey.ebrahim@gmail.com (E. Analouei Adegani),

bulboaca@math.ubbcluj.ro (T. Bulboacă)

Received: 11.04.2019; Accepted: 06.01.2020 
respectively. In particular, $\mathcal{S}^{*}:=\mathcal{S}^{*}(0)$ and $\mathcal{C}:=\mathcal{C}(0)$ are the class of starlike functions and convex functions in the unit disk $\mathbb{U}$, respectively.

Also, we denote by $\mathcal{K}(\alpha)$ the subclass of $\mathcal{A}$ consisting of functions which are close-toconvex of order $\alpha$, that is

$$
\operatorname{Re} \frac{z f^{\prime}(z)}{g(z)}>\alpha, z \in \mathbb{U},(0 \leq \alpha<1)
$$

for some function $g \in \mathcal{S}^{*}$. In particular, $\mathcal{K}:=\mathcal{K}(0)$ is the class of close-to-convex functions in the unit disk $\mathbb{U}$.

There has been a continuous interest shown on the geometric and other related properties as univalency, starlikeness, convexity, and uniformly convexity of various special functions such as Bessel, Struve, Lommel, Wright, and Bessel functions. Several authors obtained many applications in the geometric functions theory for these special functions, see for example $[1-5,7,12,13]$.

Special functions, like Bessel functions of the first kind play an important role in pure and applied mathematics. First, we will define the generalized Bessel function of first kind of order $\nu$ by

$$
J_{\nu}^{c}(z):=\sum_{n=0}^{\infty} \frac{(-c)^{n}(z / 2)^{2 n+\nu}}{n ! \Gamma(\nu+n+1)}, z \in \mathbb{U} .
$$

In the present paper we will use the following normalized form of generalized Dini function:

$$
\begin{aligned}
r_{\nu}^{c}(z) & :=2^{\nu} \Gamma(\nu+1) z^{1-\frac{\nu}{2}}\left[(1-\nu) J_{\nu}^{c}(\sqrt{z})+\sqrt{z}\left(J_{\nu}^{c}\right)^{\prime}(\sqrt{z})\right] \\
& =z+\sum_{n=1}^{\infty} \frac{(-c)^{n}(2 n+1) \Gamma(\nu+1)}{4^{n} n ! \Gamma(\nu+n+1)} z^{n+1}, z \in \mathbb{U} \quad(c \in \mathbb{C}, \nu>-1) .
\end{aligned}
$$

By taking $c=-k$, where $k>0$, we get the modified Dini function which has the form

$$
R_{\nu}^{k}(z)=z+\sum_{n=1}^{\infty} \frac{k^{n}(2 n+1) \Gamma(\nu+1)}{4^{n} n ! \Gamma(\nu+n+1)} z^{n+1}, z \in \mathbb{U} .
$$

In 2018, Bansal et al. [1] investigated some certain geometric properties of the modified Dini function $R_{\nu}^{k}$ like close-to-convexity, starlikeness, and strongly starlikeness in the open unit disk. In this paper our aim is to study some properties of the normalized form of generalized Dini function $r_{\nu}^{c}$. For this work, the following lemmas and definition will be used in our investigation.

In 2018, Bukhari et al. [6] introduced the class $\mathcal{U N}(g, \gamma, b, k)$, which was defined as follows:

Definition 1.1. Let $f \in \mathcal{A}$ be given by (1.1). Then, $f \in \mathcal{U} \mathcal{M}(g, \gamma, b, k)$ if for the function $g \in \mathcal{A}$ given by $g(z)=z+\sum_{n=2}^{\infty} b_{n} z^{n}$ with $b_{n} \geq 0$ for $n \geq 2$, we have

$$
\operatorname{Re}\left\{1+\frac{1}{b}\left(\frac{z F_{\gamma}^{\prime}(z)}{F_{\gamma}(z)}-1\right)\right\}>k\left|\frac{1}{b}\left(\frac{z F_{\gamma}^{\prime}(z)}{F_{\gamma}(z)}-1\right)\right|, z \in \mathbb{U},
$$

where

$$
\begin{aligned}
& F_{\gamma}(z):=(1-\gamma)(f * g)(z)(z)+\gamma z(f * g)^{\prime}(z)=z+\sum_{n=2}^{\infty} a_{n} d_{n}(\gamma) z^{n}, z \in \mathbb{U}, \\
& d_{n}(\gamma):=[1+n(1-\gamma)] b_{n},(k \geq 0,0 \leq \gamma \leq 1, b \in \mathbb{C} \backslash\{0\}),
\end{aligned}
$$

and "*" represents the Hadamard (or convolution) product. 
Generally, this class consists of functions $F_{\gamma}$ which are uniformly k-starlike functions of complex order $b$ in $\mathbb{U}$. For special choices of parameters of the class $\mathcal{U N}(g, \gamma, b, k)$ like $g(z)=\frac{z}{1-z}$ we obtain the subclass $\operatorname{UM}\left(\frac{z}{1-z}, \gamma, b, k\right)=: \operatorname{UMN}(\gamma, b, k)$.

The following lemmas will be used in the proofs of our main results.

Lemma 1.2. [6, Theorem 3.4] If $f \in \mathcal{A}$ be given by (1.1),

$$
g(z)=z+\sum_{n=2}^{\infty} b_{n} z^{n} \in \mathcal{A},
$$

for some $b_{k} \geq 0$, and

$$
\sum_{n=2}^{\infty}[(k+1)(n-1)+|b|][1+\gamma(n-1)] b_{n}\left|a_{n}\right|<|b|,
$$

for some $k \geq 0,0 \leq \gamma \leq 1, b \in \mathbb{C} \backslash\{0\}$, then $f \in \mathcal{U M}(g, \gamma, b, k)$.

Lemma 1.3. [16, Corollary 2] Let $\gamma \in[0,1)$. If $f \in \mathcal{A}$ satisfies the inequality

$$
\left|z f^{\prime \prime}(z)\right|<\frac{1-\gamma}{4}, z \in \mathbb{U},
$$

then

$$
\operatorname{Re} f^{\prime}(z)>\frac{1+\gamma}{2}, z \in \mathbb{U} .
$$

Letting $q \rightarrow 1^{-}$in Theorem 2.6 from [17] we obtain the next lemma.

Lemma 1.4. Let $f(z)=z+\sum_{n=1}^{\infty} b_{2 n+1} z^{2 n+1}, z \in \mathbb{U}$, be an odd function. If

$$
1 \geq 3 b_{3} \geq \ldots \geq(2 n+1) b_{2 n+1} \geq \ldots \geq 0,
$$

or

$$
1 \leq 3 b_{3} \leq \ldots \leq(2 n+1) b_{2 n+1} \leq \ldots \leq 2,
$$

then the function $f$ is close-to-convex (univalent) with respect to the convex function $\frac{1}{2} \log \frac{1+z}{1-z}$.

\section{New properties of the generalized Dini function}

Theorem 2.1. If $\gamma \in[0,1)$ and $\nu>\frac{|c|(5-2 \gamma)}{4(1-\gamma)}-1$, then $\frac{r_{\nu}^{c}(z)}{z} \in \mathcal{P}(\gamma)$.

Proof. Let define the function $p$ by

$$
p(z)=\frac{\frac{r_{\nu}^{c}(z)}{z}-\gamma}{1-\gamma}, z \in \mathbb{U} .
$$

Since $r_{\nu}^{c}$ is given by (1.2), then $p$ is analytic in $\mathbb{U}$, with $p(0)=1$. To prove our result it is sufficient to show that $|p(z)-1|<1, z \in \mathbb{U}$. By using the equality

$$
\frac{\Gamma(\nu+1)}{\Gamma(\nu+n+1)}=\frac{1}{(\nu+1)(\nu+2) \ldots(\nu+n)}=: \frac{1}{(\nu+1)_{n}}, \quad n \in \mathbb{N},
$$

the inequalities

$$
4^{n} \geq \frac{4}{3}(2 n+1), \quad(\nu+1)_{n} \geq(\nu+1)^{n}, \quad n ! \geq 2^{n-1}, \quad n \in \mathbb{N},
$$


and the well-known triangle inequality, we deduce that

$$
\begin{aligned}
& |p(z)-1|=\left|\frac{1}{1-\gamma} \sum_{n=1}^{\infty} \frac{(-c)^{n}(2 n+1) \Gamma(\nu+1)}{4^{n} n ! \Gamma(\nu+n+1)} z^{n}\right| \\
& \leq \frac{1}{1-\gamma} \sum_{n=1}^{\infty} \frac{|c|^{n}(2 n+1) \Gamma(\nu+1)}{4^{n} n ! \Gamma(\nu+n+1)}|z|^{n} \leq \frac{3}{4(1-\gamma)} \sum_{n=1}^{\infty} \frac{|c|^{n} \Gamma(\nu+1)}{2^{n-1} \Gamma(\nu+n+1)} \\
& =\frac{3}{2(1-\gamma)} \sum_{n=1}^{\infty} \frac{|c|^{n}}{2^{n}(\nu+1)_{n}} \leq \frac{3}{2(1-\gamma)} \sum_{n=1}^{\infty}\left(\frac{|c|}{2(\nu+1)}\right)^{n}, z \in \mathbb{U} .
\end{aligned}
$$

Using the fact that the assumption $\nu>\frac{|c|(5-2 \gamma)}{4(1-\gamma)}-1$ implies $\frac{|c|}{2(\nu+1)}<1$, from the above inequality it follows that

$$
|p(z)-1| \leq \frac{3}{2(1-\gamma)} \sum_{n=1}^{\infty}\left(\frac{|c|}{2(\nu+1)}\right)^{n}=\frac{1}{1-\gamma} \frac{3|c|}{4(\nu+1)-2|c|}:=\mu, z \in \mathbb{U} .
$$

Under our hypothesis, it is easy to check that $\mu<1$, and therefore $\frac{r_{\nu}^{c}(z)}{z} \in \mathcal{P}(\gamma)$.

For $\gamma=0$ the Theorem 2.1 reduces to the following result which gives sufficient condition for the function $\frac{r_{\nu}^{c}(z)}{z}$ to be in the class $\mathcal{P}$.

Corollary 2.2. If $\nu>\frac{5|c|}{4}-1$, then $\frac{r_{\nu}^{c}(z)}{z} \in \mathcal{P}$.

Example 2.3. Since

$$
J_{\frac{1}{2}}^{1}(z)=\sqrt{\frac{2}{\pi z}} \sin z,
$$

from Corollary 2.2 it follows that the function

$$
\frac{r_{\frac{1}{2}}^{1}(z)}{z}=\cos \sqrt{z}
$$

is in the class $\mathcal{P}$, and thus

$$
\operatorname{Re}(\cos \sqrt{z})>0, z \in \mathbb{U} \text {. }
$$

Theorem 2.4. If $\gamma \in[0,1)$ and $\nu>\frac{|c|(7-\gamma+2 \sqrt{12-3 \gamma})}{2(1-\gamma)}-1$, then $r_{\nu}^{c} \in \mathcal{K}\left(\frac{1+\gamma}{2}\right)$.

Proof. Using the relation (2.1), the inequalities

$$
4^{n} \geq \frac{2}{3}(n+1)(2 n+1), \quad(\nu+1)_{n} \geq(\nu+1)^{n}, \quad n ! \geq 2^{n-1}, \quad n \in \mathbb{N},
$$

and the triangle inequality, we have

$$
\begin{aligned}
& \left|z\left(r_{\nu}^{c}\right)^{\prime \prime}(z)\right|=\left|\sum_{n=1}^{\infty} \frac{(-c)^{n} n(n+1)(2 n+1) \Gamma(\nu+1)}{4^{n} n ! \Gamma(\nu+n+1)} z^{n}\right| \\
& \leq \sum_{n=1}^{\infty} \frac{|c|^{n} n(n+1)(2 n+1) \Gamma(\nu+1)}{4^{n} n ! \Gamma(\nu+n+1)}|z|^{n} \leq \frac{3}{2} \sum_{n=1}^{\infty} \frac{|c|^{n} n \Gamma(\nu+1)}{2^{n-1} \Gamma(\nu+n+1)} \\
& =\frac{3}{2} \sum_{n=1}^{\infty} \frac{n|c|^{n}}{2^{n-1}(\nu+1)_{n}} \leq \frac{3}{2} \sum_{n=1}^{\infty} \frac{n|c|^{n}}{2^{n-1}(\nu+1)^{n}}=\frac{3}{2} \frac{|c|}{\nu+1} \sum_{n=1}^{\infty} n\left(\frac{|c|}{2(\nu+1)}\right)^{n-1}, z \in \mathbb{U} .
\end{aligned}
$$


From the assumption $\nu>\frac{|c|(7-\gamma+2 \sqrt{12-3 \gamma})}{2(1-\gamma)}-1$ it follows that $\frac{|c|}{2(\nu+1)}<1$, and from the above inequality we deduce

$$
\begin{aligned}
& \left|z\left(r_{\nu}^{c}\right)^{\prime \prime}(z)\right| \leq \frac{3}{2} \frac{|c|}{\nu+1} \sum_{n=1}^{\infty} n\left(\frac{|c|}{2(\nu+1)}\right)^{n-1} \\
& =\frac{3}{2} \frac{|c|}{\nu+1} \frac{4(\nu+1)^{2}}{[2(\nu+1)-|c|]^{2}}=\frac{6|c|(\nu+1)}{[2(\nu+1)-|c|]^{2}}:=\lambda, z \in \mathbb{U} .
\end{aligned}
$$

It is easy to check that our assumption implies that $\lambda<\frac{1-\gamma}{4}$ thus, from the previous inequality we obtain that

$$
\left|z\left(r_{\nu}^{c}\right)^{\prime \prime}(z)\right|<\frac{1-\gamma}{4}, z \in \mathbb{U} .
$$

Now, by using Lemma 1.3 we conclude that

$$
\operatorname{Re}\left(r_{\nu}^{c}\right)^{\prime}(z)>\frac{1+\gamma}{2}, z \in \mathbb{U}
$$

and therefore $r_{\nu}^{c} \in \mathcal{K}\left(\frac{1+\gamma}{2}\right)$.

For the special case $\gamma=0$ the Theorem 2.4 leads to the following result which gives sufficient condition for the function $r_{\nu}^{c}$ to be of close-to-convex of order $\frac{1}{2}$.

Corollary 2.5. If $\nu>\frac{|c|(7+4 \sqrt{3})}{2}-1$, then $r_{\nu}^{c} \in \mathcal{K}\left(\frac{1}{2}\right)$.

The following result gives us a sufficient conditions for the function $r_{\nu}^{c}$ to be in the class $\operatorname{UM}(\gamma, b, k):=\operatorname{UM}\left(\frac{z}{1-z}, \gamma, b, k\right)$.

Theorem 2.6. If

$$
\gamma(k+1)\left(r_{\nu}^{c}\right)^{\prime \prime}(1)+[k+1+\gamma(|b|-(k+1))]\left(r_{\nu}^{c}\right)^{\prime}(1)+(|b|-(k+1))(1-\gamma) r_{\nu}^{c}(1)<2|b|,
$$

then the function $r_{\nu}^{c} \in \mathcal{U M}(\gamma, b, k)$.

Proof. To prove our result, since $r_{\nu}^{c}(z)=z+\sum_{n=2}^{\infty} a_{n} z^{n}$ with $a_{n}=\frac{(-c)^{n-1}(2 n-1) \Gamma(\nu+1)}{4^{n-1}(n-1) ! \Gamma(\nu+n)}$, according to Lemma 1.2 it is sufficient to show that

$$
\sum_{n=2}^{\infty}[(k+1)(n-1)+|b|][1+\gamma(n-1)]\left|a_{n}\right|<|b| .
$$

Using the assumption (2.2) it is easy to prove that

$$
\begin{aligned}
& \sum_{n=2}^{\infty}[(k+1)(n-1)+|b|][1+\gamma(n-1)]\left|a_{n}\right|=\sum_{n=2}^{\infty} n[k+1+\gamma(|b|-(k+1))]\left|a_{n}\right| \\
& +\sum_{n=2}^{\infty} n(n-1) \gamma(k+1)\left|a_{n}\right|+\sum_{n=2}^{\infty}(|b|-(k+1))(1-\gamma)\left|a_{n}\right| \\
& =[k+1+\gamma(|b|-(k+1))]\left[\left(r_{\nu}^{c}\right)^{\prime}(1)-1\right]+\gamma(k+1)\left(r_{\nu}^{c}\right)^{\prime \prime}(1) \\
& +(|b|-(k+1))(1-\gamma)\left(r_{\nu}^{c}(1)-1\right)=\gamma(k+1)\left(r_{\nu}^{c}\right)^{\prime \prime}(1) \\
& +[k+1+\gamma(|b|-(k+1))]\left(r_{\nu}^{c}\right)^{\prime}(1)+(|b|-(k+1))(1-\gamma)\left(r_{\nu}^{c}\right)(1)-|b|<|b|,
\end{aligned}
$$

hence (2.3) holds, and consequently $r_{\nu}^{c} \in \mathcal{U N}(\gamma, b, k)$. 
Remark 2.7. We will emphasize a few special cases of the above theorem obtained for different choices of the parameters $b, k$, and $\gamma$.

(i) Letting $b=k=1$ and $\gamma=0$ in Theorem 2.6 we get: if

$$
2\left(r_{\nu}^{c}\right)^{\prime}(1)-r_{\nu}^{c}(1)<2,
$$

then the function $r_{\nu}^{c}$ belongs to the class $\mathcal{U N}(0,1,1)$ of uniformly starlike functions defined and investigated by Goodman $[8,9]$.

(ii) For $b=k=1$ and $\gamma=1$, Theorem 2.6 yields to the next result: if

$$
2\left(r_{\nu}^{c}\right)^{\prime \prime}(1)+\left(r_{\nu}^{c}\right)^{\prime}(1)<2,
$$

then the function $r_{\nu}^{c}$ belongs to the class $\mathcal{U} \mathcal{M}(1,1,1)$ of uniformly convex functions defined and studied by Goodman $[8,9]$.

(iii) By taking $b=1-\alpha$ and $\gamma=0$ in Theorem 2.6 we obtain: if

$$
(k+1)\left(r_{\nu}^{c}\right)^{\prime}(1)-(\alpha+k) r_{\nu}^{c}(1)<2(1-\alpha),
$$

then the function $r_{\nu}^{c}$ belongs to the class $\mathcal{U N}(0,1-\alpha, k)$ of k-uniformly starlike functions of order $\alpha$ introduced and investigated by Rønning [14,15].

(iv) Letting $b=1-\alpha$ and $\gamma=1$ in Theorem 2.6 we have: if

$$
(k+1)\left(r_{\nu}^{c}\right)^{\prime \prime}(1)+(1-\alpha)\left(r_{\nu}^{c}\right)^{\prime}(1)<2(1-\alpha),
$$

then the function $r_{\nu}^{c}$ belongs to the class $\mathcal{U N}(1,1-\alpha, k)$ of k-uniformly convex functions of order $\alpha$ introduced and studied by Rönning $[14,15]$.

(v) Taking $b=1$ and $\gamma=0$ in Theorem 2.6 we obtain: if

$$
(k+1)\left(r_{\nu}^{c}\right)^{\prime}(1)-k r_{\nu}^{c}(1)<2,
$$

then the function $r_{\nu}^{c}$ belongs to the class $\mathcal{U M}(0,1, k)$ of k-uniformly starlike functions investigated by Kanas and Wiśniowska [11].

(vi) Letting $b=1$ and $\gamma=1$ in Theorem 2.6 we obtain: if

$$
(k+1)\left(r_{\nu}^{c}\right)^{\prime \prime}(1)+\left(r_{\nu}^{c}\right)^{\prime}(1)<2,
$$

then the function $r_{\nu}^{c}$ is in the class $\mathcal{U N}(1,1, k)$ of k-uniformly convex functions studied by Kanas and Wiśniowska [10].

Theorem 2.8. If $\nu \geq \frac{9 k}{4}-1$, then the odd function $\frac{R_{\nu}^{k}\left(z^{2}\right)}{z}$ is close-to-convex with respect to the convex function $\frac{1}{2} \log \frac{1+z}{1-z}$.

Proof. Since $\frac{R_{\nu}^{k}\left(z^{2}\right)}{z}=\sum_{n=1}^{\infty} d_{2 n-1} z^{2 n-1}$, where

$$
d_{2 n-1}=\frac{k^{n-1}(2 n-1) \Gamma(\nu+1)}{4^{n-1}(n-1) ! \Gamma(\nu+n)},
$$

then $d_{2 n-1}>0$ for all $n \geq 1$. In view of Lemma 1.4, to prove our result it is sufficient to show that $\left\{(2 n-1) d_{2 n-1}\right\}_{n>1}$ is a non-increasing sequence.

First, for $n=2$, the assumption $\nu \geq \frac{9 k}{4}-1$ implies that

$$
3 d_{3}=\frac{9 k}{4(\nu+1)} \leq 1
$$


If $n \geq 2$, a simple computation shows that

$$
\begin{aligned}
& (2 n-1) d_{2 n-1}-(2 n+1) d_{2 n+1}=\frac{k^{n-1} \Gamma(\nu+1)}{4^{n-1}(n-1) ! \Gamma(\nu+n)}\left[(2 n-1)^{2}-\frac{k(2 n+1)^{2}}{4 n(\nu+n)}\right] \\
& =\frac{k^{n-1} \Gamma(\nu+1)}{4^{n} n ! \Gamma(\nu+n)}\left[4 n(2 n-1)^{2}(\nu+n)-k(2 n+1)^{2}\right]=\frac{k^{n-1} \Gamma(\nu+1)}{4^{n} n ! \Gamma(\nu+n)} \varphi(n),
\end{aligned}
$$

where

$$
\varphi(n):=4 n(2 n-1)^{2}(\nu+n)-k(2 n+1)^{2} .
$$

Thus, it is sufficient to prove $\varphi(n) \geq 0$ for all $n \geq 2$. Using the inequality

$$
4 n(2 n-1)^{2} \geq(2 n+1)^{2}, n \geq 2,
$$

we have

$$
\begin{aligned}
& \varphi(n)=4 n(2 n-1)^{2}(\nu+n)-k(2 n+1)^{2} \geq 4 n(2 n-1)^{2}(\nu+2)-k(2 n+1)^{2} \\
& \geq(2 n+1)^{2}(\nu+2)-k(2 n+1)^{2}=(2 n+1)^{2}(\nu+2-k) \geq(2 n+1)^{2}\left(\frac{5 k}{4}+1\right)>0,
\end{aligned}
$$

whenever $\nu \geq \frac{9 k}{4}-1$.

Therefore, $\left\{(2 n-1) c_{2 n-1}\right\}_{n \geq 1}$ is a non-increasing sequence and from Lemma 1.4 our result follows.

Remark 2.9. Similar results to Theorems 2.1 and 2.8 can be found for the generalized Dini functions $d_{\nu, a, b, c}$ in Theorem 6 (iv) and Theorem 18 in [7].

\section{References}

[1] D. Bansal, M.K. Soni and A. Soni, Certain geometric properties of the modified Dini function, Anal. Math. Phys. 9, 1383-1392, 2019.

[2] Á. Baricz, E. Deniz and N. Yagmur, Close-to-convexity of normalized Dini functions, Math. Nachr. 289, 1721-1726, 2016.

[3] Á. Baricz, P.A. Kupán and R. Szász, The radius of starlikeness of normalized Bessel functions of the first kind, Proc. Amer. Math. Soc. 142 (6), 2019-2025, 2014.

[4] Á. Baricz, S. Ponnusamy and S. Singh, Modified Dini functions: monotonicity patterns and functional inequalities, Acta Math. Hungar. 149 (1), 120-142, 2016.

[5] Á. Baricz, E. Toklu and E. Kadioğlu, Radii of starlikeness and convexity of Wright functions, Math. Commun. 23 (1), 97-117, 2018.

[6] S.Z.H. Bukhari, J. Sokól and S. Zafar, Unified approach to starlike and convex functions involving convolution between analytic functions, Results Math. 73, Article number: 30, 2018.

[7] M.U. Din, M. Raza, S. Hussain and M. Darus, Certain geometric properties of generalized Dini functions, J. Funct. Spaces, 2018, Art. ID 2684023, 1-9, 2018.

[8] A.W. Goodman, On uniformly convex functions, Ann. Polon. Math. 56, 87-92, 1991.

[9] A.W. Goodman, On uniformly starlike functions, Ann. Polon. Math. 155, 364-370, 1991.

[10] S. Kanas and A. Wiśniowska, Conic regions and k-uniform convexity, J. Comput. Appl. Math. 105, 327-336, 1999.

[11] S. Kanas and A. Wiśniowska, Conic regions and k-starlike functions, Rev. Roumaine Math. Pures Appl. 45 (4), 647-657, 2000.

[12] J.K. Prajapat, Certain geometric properties of the Wright functions, Integral Transforms Spec. Funct. 26 (3), 203-212, 2015. 
[13] D. Răducanu, Geometric properties of Mittag-Leffler functions, in: Models and Theories in Social Systems, Studies in Systems, Decision and Control 79, 403-415, Springer, Cham, 2018.

[14] F. Rönning, On starlike functions associated with parabolic regions, Ann. Univ. Mariae Curie-Sklodowska Sect. A, 45, 117-122, 1991.

[15] F. Rönning, Uniformly convex functions and a corresponding class of starlike functions. Proc. Amer. Math. Soc. 1 (18), 189-196, 1993.

[16] S. Owa, M. Nunokawa, H. Saitoh and H.M. Srivastava, Close-to-convexity, starlikeness and convexity of certain analytic functions, Appl. Math. Lett. 15, 63-69, 2002.

[17] S.K. Sahoo and N.L. Sharma, On a generalization of close-to-convex functions, Ann. Polon. Math. 113 (1), 93-108, 2015. 